\title{
THE SYMMETRIC GROUP REPRESENTATION ON COHOMOLOGY OF THE REGULAR ELEMENTS OF A MAXIMAL TORUS OF THE SPECIAL LINEAR GROUP
}

\author{
ANTHONY HENDERSON
}

(Received 22 November 2006; revised 20 June 2007)

Communicated by J. Du

\begin{abstract}
We give a formula for the character of the representation of the symmetric group $S_{n}$ on each isotypic component of the cohomology of the set of regular elements of a maximal torus of $S L_{n}$, with respect to the action of the centre.
\end{abstract}

2000 Mathematics subject classification: 20C30, 58A10.

Keywords and phrases: symmetric group, representation, cohomology.

\section{Introduction}

Let $n$ be a positive integer. Define the hyperplane complement

$$
T(1, n):=\left\{\left(z_{1}, z_{2}, \ldots, z_{n}\right) \in \mathbb{C}^{n} \mid z_{i} \neq 0, \forall i, z_{i} \neq z_{j}, \forall i \neq j\right\} .
$$

The symmetric group $S_{n}$ acts on $T(1, n)$ by permuting coordinates; we can identify $T(1, n)$ with the set of regular elements of a maximal torus of $G L_{n}(\mathbb{C})$, and $S_{n}$ with the Weyl group of the maximal torus. This action induces representations of $S_{n}$ on the cohomology groups $H^{i}(T(1, n))$ (taken with complex coefficients). The characters of these representations are well known (they will be encapsulated in a single 'equivariant generating function' in Theorem 3.1 below). For the purposes of this introduction, we recall only the 'nonequivariant' information, that is, the Betti numbers of $T(1, n)$ :

$$
\sum_{i}(-1)^{i} \operatorname{dim} H^{i}(T(1, n)) q^{n-i}=(q-1)(q-2) \cdots(q-n) .
$$

These Betti numbers are particularly familiar, since $T(1, n)$ is homotopy equivalent to the configuration space $C_{n+1}$ of $(n+1)$-tuples of distinct complex numbers.

This work was supported by Australian Research Council grant DP0344185.

(C) 2008 Australian Mathematical Society 1446-7887/08 \$A2.00+0.00 
Now consider the toral complement

$$
S T(1, n):=\left\{\left(z_{1}, z_{2}, \ldots, z_{n}\right) \in T(1, n) \mid z_{1} z_{2} \cdots z_{n}=1\right\} .
$$

This can be identified with the set of regular elements of a maximal torus of $S L_{n}(\mathbb{C})$. Of course $S_{n}$ still acts, and there is a commuting action of $\mu_{n}$ (the centre of $S L_{n}(\mathbb{C})$ ) by scaling. Thus we have a direct sum decomposition of $S_{n}$-representations:

$$
H^{i}(S T(1, n)) \cong \bigoplus_{\chi \in \widehat{\mu_{n}}} H^{i}(S T(1, n))_{\chi}
$$

where $H^{i}(S T(1, n))_{\chi}$ is the $\chi$-isotypic component of $H^{i}(S T(1, n))$. In this paper we address the following problem, suggested by Lehrer.

PRoblem 1.1. Give a formula for the character of the representation of $S_{n}$ on each $H^{i}(S T(1, n))_{\chi}$.

Our solution is given in Section 3 (see especially (3.6)). For now, we state merely the nonequivariant version:

$$
\begin{aligned}
& \sum_{i}(-1)^{i} \operatorname{dim} H^{i}(S T(1, n))_{\chi} q^{n-1-i} \\
& \quad=\frac{(-1)^{n-n / r} n !}{r^{n / r}(n / r) !}(q-r-1)(q-2 r-1) \cdots\left(q-\left(\frac{n}{r}-1\right) r-1\right),
\end{aligned}
$$

where $r$ is the order of $\chi$ in the character group $\widehat{\mu_{n}}$.

Summing over $\chi$, we deduce a formula (3.7) for the character of the total representation on $H^{i}(S T(1, n))$, of which the nonequivariant specialization is

$$
\begin{aligned}
& \sum_{i}(-1)^{i} \operatorname{dim} H^{i}(S T(1, n)) q^{n-1-i} \\
& \quad=\sum_{r \mid n} \frac{(-1)^{n-n / r} \phi(r) n !}{r^{n / r}(n / r) !}(q-r-1)(q-2 r-1) \cdots\left(q-\left(\frac{n}{r}-1\right) r-1\right) .
\end{aligned}
$$

This total formula was essentially already known. Since $S T(1, n)$ is 'minimally pure' in the sense of [2], (1.4) follows from the fact that the right-hand side counts the number of $\mathbb{F}_{q}$-points of the variety $S T(1, n)$ for all prime powers $q$ which are congruent to $1 \bmod n$. More generally, (3.7) follows from a count of fixed points of twisted Frobenius maps, which was the result [3, Theorem 5.8] of Fleischmann and Janiszczak. See Remark 3.4 below.

Clearly the quotient of $S T(1, n)$ by $\mu_{n}$ can be identified with $\mathbb{P} T(1, n)$, the image of $T(1, n)$ in $\mathbb{P}^{n-1}(\mathbb{C})$. So, in the case where $\chi$ is the trivial character, we are dealing with

$$
H^{i}(S T(1, n))^{\mu_{n}} \cong H^{i}(\mathbb{P} T(1, n)) .
$$

In general, as (1.3) suggests, the representation $H^{i}(S T(1, n))_{\chi}$ is induced from the wreath product subgroup $W(r, n / r):=\mu_{r} 2 S_{n / r}$ of $S_{n}$. To be precise, define the hyperplane complement 


$$
T(r, m):=\left\{\left(z_{1}, z_{2}, \ldots, z_{m}\right) \in \mathbb{C}^{m} \mid z_{i} \neq 0, \forall i, z_{i}^{r} \neq z_{j}^{r}, \forall i \neq j\right\}
$$

and its image $\mathbb{P} T(r, m)$ in $\mathbb{P}^{m-1}(\mathbb{C})$. For $r \geq 2$, we identify the corresponding reflection group $G(r, 1, m)$ with the wreath product $W(r, m)$ (if $r=1, W(1, m)=S_{m}$ acts on $T(1, m)$ as seen above). In the following theorem, $\varepsilon_{n}$ denotes the sign character of $S_{n}$, and $\operatorname{det}_{n / r}$ the determinant character of $G L_{n / r}(\mathbb{C})$, restricted to $W(r, n / r)$.

THEOREM 1.2. Let $r$ be the order of $\chi \in \widehat{\mu_{n}}$. For every $i$, we have an isomorphism of representations of $S_{n}$ :

$$
H^{i}(S T(1, n))_{\chi} \cong \varepsilon_{n} \otimes \operatorname{Ind}_{W(r, n / r)}^{S_{n}}\left(\operatorname{det}_{n / r} \otimes H^{i-n+n / r}(\mathbb{P} T(r, n / r))\right) .
$$

The proof of this theorem given in Section 4 merely equates the characters of both sides; a more conceptual understanding of the isomorphism (or rather the related isomorphism (4.2)), involving Orlik-Solomon-style bases for the cohomology groups, is given in [6].

When $\chi$ is faithful (that is, $r=n$ ), Theorem 1.2 says that

$$
H^{i}(S T(1, n))_{\chi} \cong \begin{cases}\varepsilon_{n} \otimes \operatorname{Ind}_{\mu_{n}}^{S_{n}}(\psi) & \text { if } i=n-1 \\ 0 & \text { otherwise }\end{cases}
$$

where $\mu_{n}$ is embedded in $S_{n}$ as the subgroup generated by an $n$-cycle, and $\psi \in \widehat{\mu_{n}}$ is any faithful character (it does not matter which-note also that tensoring with $\varepsilon_{n}$ makes no difference unless $n \equiv 2(\bmod 4))$. This result for prime $n$ was proved in [2, Section 4.4].

\section{Equivariant weight polynomials}

Suppose that $X$ is an irreducible complex variety which is minimally pure in the sense that $H_{c}^{i}(X)$ is a pure Hodge structure of weight $2 i-2 \operatorname{dim} X$ for all $i$ (see [2]). Let $\Gamma$ be a finite group acting on $X$. We define the equivariant weight polynomials of this action by

$$
P(\gamma, X, q):=\sum_{i}(-1)^{i} \operatorname{tr}\left(\gamma, H_{c}^{i}(X)\right) q^{i-\operatorname{dim} X},
$$

for all $\gamma \in \Gamma$, where $q$ is an indeterminate ( $=t^{2}$ in the notation of [2]). We also define

$$
P^{\Gamma}(X, q):=\sum_{i}(-1)^{i}\left[H_{c}^{i}(X)\right] q^{i-\operatorname{dim} X} \in R(\Gamma)[q],
$$

where $R(\Gamma)$ is the complexified representation ring of $\Gamma$. If $\Delta$ is an abelian finite group acting on $X$ whose action commutes with that of $\Gamma$, and $\chi$ is a character of $\Delta$, we define 


$$
\begin{aligned}
P(\gamma, \chi, X, q) & :=\sum_{i}(-1)^{i} \operatorname{tr}\left(\gamma, H_{c}^{i}(X)_{\chi}\right) q^{i-\operatorname{dim} X} \\
& =\sum_{i}(-1)^{i} \frac{1}{|\Delta|} \sum_{\delta \in \Delta} \chi(\delta)^{-1} \operatorname{tr}\left((\gamma, \delta), H_{c}^{i}(X)\right) q^{i-\operatorname{dim} X} \\
& =\frac{1}{|\Delta|} \sum_{\delta \in \Delta} \chi(\delta)^{-1} P((\gamma, \delta), X, q),
\end{aligned}
$$

and similarly

$$
P^{\Gamma}(\chi, X, q):=\sum_{i}(-1)^{i}\left[H_{c}^{i}(X)_{\chi}\right] q^{i-\operatorname{dim} X} \in R(\Gamma)[q] .
$$

If $X$ is nonsingular, we can translate knowledge of $H_{c}^{i}(X)$ and $H_{c}^{i}(X)_{\chi}$ into knowledge of $H^{i}(X)$ and $H^{i}(X)_{\chi}$ by Poincaré duality.

Now for any positive integers $r$ and $n, T(r, n)$ (respectively, $\mathbb{P} T(r, n)$ ) is a nonsingular irreducible minimally pure variety of dimension $n$ (respectively, $n-1$ ); minimal purity is a standard property of hyperplane complements [2, Example 3.3]. Also, $S T(1, n)$ is clearly a nonsingular irreducible variety of dimension $n-1$. To show that it is minimally pure, one can use [2, Corollary 4.2], or else observe that it is the quotient of $\mathbb{P} T(n, n)$ by the free action of a finite group, as follows.

Recall that $W(r, m)=S_{m} \ltimes \mu_{r}^{m}$ acts on $T(r, m)$ and $\mathbb{P} T(r, m)$; the $S_{m}$ factor acts by permuting the coordinates, and $\mu_{r}^{m}$ acts by scaling them. Define a surjective map $\varphi: \mathbb{P} T(n, n) \rightarrow S T(1, n)$ by

$$
\varphi\left(\left[x_{1}: x_{2}: \cdots: x_{n}\right]\right)=\frac{1}{x_{1} x_{2} \cdots x_{n}}\left(x_{1}^{n}, x_{2}^{n}, \ldots, x_{n}^{n}\right) .
$$

The fibres of $\varphi$ are clearly the orbits of the normal subgroup $S \mu_{n}^{n} \subset W(n, n)$, where

$$
S \mu_{n}^{m}:=\left\{\left(\zeta_{1}, \ldots, \zeta_{m}\right) \in \mu_{n}^{m} \mid \zeta_{1} \zeta_{2} \cdots \zeta_{m}=1\right\} .
$$

The action of $S \mu_{n}^{n}$ on $\mathbb{P} T(n, n)$ becomes free once one factors out the subgroup $\{(\zeta, \zeta, \ldots, \zeta)\}$, which acts trivially. Thus $S T(1, n)$ is minimally pure, and solving Problem 1.1 amounts to computing the polynomials $P(w, \chi, S T(1, n), q)$, for all $w \in S_{n}$ and $\chi \in \widehat{\mu_{n}}$.

Consider the quotient of $T(n, m)$ by $S \mu_{n}^{m}$ for arbitrary $m \geq 1$. This can be identified with

$$
T^{(n)}(1, m):=\left\{\left(\left(z_{i}\right), z\right) \in T(1, m) \times \mathbb{C}^{\times} \mid z^{n}=z_{1} \cdots z_{m}\right\} .
$$

The quotient map $\psi: T(n, m) \rightarrow T^{(n)}(1, m)$ is given by

$$
\psi\left(x_{1}, x_{2}, \ldots, x_{m}\right)=\left(\left(x_{1}^{n}, x_{2}^{n}, \ldots, x_{m}^{n}\right), x_{1} x_{2} \cdots x_{m}\right) .
$$

The group $S_{m} \times \mu_{n} \cong W(n, m) / S \mu_{n}^{m}$ acts on the quotient $T^{(n)}(1, m)$ in the obvious way: $S_{m}$ acts on the $T(1, m)$ component, and $\mu_{n}$ acts by scaling $z$. 
When $m=n$, we have an isomorphism

$$
\begin{aligned}
T^{(n)}(1, n) & \stackrel{\sim}{\rightarrow} S T(1, n) \times \mathbb{C}^{\times}, \\
\left(\left(z_{1}, \ldots, z_{n}\right), z\right) & \mapsto\left(\left(z_{1} z^{-1}, \ldots, z_{n} z^{-1}\right), z\right),
\end{aligned}
$$

which respects the $S_{n}$-action, and transforms the $\mu_{n}$-action on $T^{(n)}(1, n)$ into the inverse of the $\mu_{n}$-action on $S T(1, n)$, and a scaling action on $\mathbb{C}^{\times}$. Since the latter has no effect on cohomology,

$$
P(w, \chi, S T(1, n), q)=\frac{1}{q-1} P\left(w, \chi^{-1}, T^{(n)}(1, n), q\right) .
$$

So we aim to compute $P\left(w, \chi^{-1}, T^{(n)}(1, n), q\right)$; it turns out to be convenient to compute the polynomials $P\left(w, \chi^{-1}, T^{(n)}(1, m), q\right)$ for all $m \geq 1$ and $w \in S_{m}$ together.

REMARK 2.1. One can see $a$ priori that allowing $m \neq n$ incurs no extra work, thanks to the following neat $\operatorname{argument}$, pointed out by Lehrer. If $d=\operatorname{gcd}(m, n)$, the action of $\mu_{n / d} \subset \mu_{n}$ on $T^{(n)}(1, m)$ is part of the action of the connected group $\mathbb{C}^{\times}$defined by

$$
t \cdot\left(\left(z_{i}\right), z\right)=\left(\left(t^{n / d} z_{i}\right), t^{m / d} z\right) .
$$

Hence $\mu_{n / d}$ acts trivially on cohomology, so $P\left(w, \chi^{-1}, T^{(n)}(1, m), q\right)=0$ unless $\left.\chi\right|_{\mu_{n / d}}=1$, that is, $\chi^{d}=1$, or $r \mid m$, where $r$ is the order of $\chi$. Moreover, if $r \mid m$, then writing $\chi^{\circ}$ for the character of $\mu_{r}$ such that $\chi(\zeta)=\chi^{\circ}\left(\zeta^{n / r}\right)$ for all $\zeta \in \mu_{n}$, and $\chi^{\prime}$ for the character of $\mu_{m}$ defined by $\chi^{\prime}(\zeta)=\chi^{\circ}\left(\zeta^{m / r}\right)$ for all $\zeta \in \mu_{m}$,

$$
\begin{aligned}
P\left(w, \chi^{-1}, T^{(n)}(1, m), q\right) & =P\left(w,\left(\chi^{\circ}\right)^{-1}, T^{(r)}(1, m), q\right) \\
& =P\left(w,\left(\chi^{\prime}\right)^{-1}, T^{(m)}(1, m), q\right) .
\end{aligned}
$$

We will not actually use this observation.

The identification of $T^{(n)}(1, m)$ with the quotient of $T(n, m)$ by $S \mu_{n}^{m}$ has the following consequence for equivariant weight polynomials.

Proposition 2.2. For any $w \in S_{m}$ and $\chi \in \widehat{\mu_{n}}$,

$$
\begin{aligned}
P\left(w, \chi^{-1}, T^{(n)}(1, m), q\right) & \\
& =\frac{1}{n^{m}} \sum_{\left(\zeta_{i}\right) \in \mu_{n}^{m}} \chi\left(\zeta_{1} \cdots \zeta_{m}\right) P\left(w\left(\zeta_{1}, \ldots, \zeta_{m}\right), T(n, m), q\right) .
\end{aligned}
$$

PROOF. It is well known that if $V$ is a representation of the finite group $G$ and $V^{H}$ is the subspace invariant under the normal subgroup $H$, the character of $G / H$ on $V^{H}$ is given by

$$
\operatorname{tr}\left(g H, V^{H}\right)=\frac{1}{|H|} \sum_{h \in H} \operatorname{tr}(g h, V)
$$


Now apply this with $V=H_{c}^{i}(T(n, m)), G=W(n, m)$, and $H=S \mu_{n}^{m}$, so that $V^{H} \cong$ $H_{c}^{i}\left(T^{(n)}(1, m)\right)$ and $G / H \cong S_{m} \times \mu_{n}$. We find that, for all $\zeta \in \mu_{n}$,

$$
P\left((w, \zeta), T^{(n)}(1, m), q\right)=\frac{1}{n^{m-1}} \sum_{\substack{\left(\zeta_{i}\right) \in \mu_{n}^{m} \\ \zeta_{1} \cdots \zeta_{m}=\zeta}} P\left(w\left(\zeta_{1}, \ldots, \zeta_{m}\right), T(n, m), q\right)
$$

Combining this with the fact that

$$
P\left(w, \chi^{-1}, T^{(n)}(1, m), q\right)=\frac{1}{n} \sum_{\zeta \in \mu_{n}} \chi(\zeta) P\left((w, \zeta), T^{(n)}(1, m), q\right)
$$

gives the desired result.

\section{Generating functions}

In this section we will compute the sum in Proposition 2.2 using the known formula for the equivariant weight polynomials of $T(r, m)$. As is usual in dealing with characters of symmetric groups and wreath products, the computations become easier if one uses suitable 'equivariant generating functions'.

For any $r \geq 1$, let $\Lambda(r)$ denote the polynomial ring $\mathbb{C}\left[p_{i}(\zeta)\right]$ in countably many independent variables $p_{i}(\zeta)$ where $i$ is a positive integer and $\zeta \in \mu_{r}$. Define an $\mathbb{N}$ grading on $\Lambda(r)$ by $\operatorname{deg}\left(p_{i}(\zeta)\right)=i$. Also let $\Lambda(r)[q]:=\Lambda(r) \otimes_{\mathbb{C}} \mathbb{C}[q]$, with the $\mathbb{N}$-grading given by the first factor $(\operatorname{so} \operatorname{deg}(q)=0)$. Let $\mathbb{A}(r)=\mathbb{C} \llbracket p_{i}(\zeta) \rrbracket$ be the completion of $\Lambda(r)$, and set $\mathbb{A}(r)[q]=\mathbb{A}(r) \otimes \mathbb{C}[q]$.

As in [10, Ch. I, Appendix B], we define an isomorphism $\operatorname{ch}_{W(r, m)}: R(W(r, m)) \stackrel{\sim}{\rightarrow}$ $\Lambda(r)_{m}$ by

$$
\operatorname{ch}_{W(r, m)}([M])=\frac{1}{r^{m} m !} \sum_{y \in W(r, m)} \operatorname{tr}(y, M) p_{y},
$$

where $p_{y}=\prod_{i, \zeta} p_{i}(\zeta)^{a_{i}(\zeta)}$ if $y$ has $a_{i}(\zeta)$ cycles of length $i$ and type $\zeta$. Note that to recover $\operatorname{tr}(y, M)$ from $\operatorname{ch}_{W(r, m)}([M])$ one must multiply the coefficient of $\prod_{i, \zeta} p_{i}(\zeta)^{a_{i}(\zeta)}$ by the order of the centralizer of $y$, which is $\prod_{i, \zeta} a_{i}(\zeta) !(r i)^{a_{i}(\zeta)}$. Write $\operatorname{ch}_{W(r, m)}$ also for the induced isomorphism $R(W(r, m))[q] \stackrel{\sim}{\rightarrow} \Lambda(r)[q]_{m}$.

The result we need on the equivariant weight polynomials for $T(r, m)$ can be conveniently stated in terms of the equivariant generating function $P(r, q) \in \mathbb{A}(r)[q]$ defined by

$$
\begin{aligned}
P(r, q) & :=1+\sum_{m \geq 1} \operatorname{ch}_{W(r, m)}\left(P^{W(r, m)}(T(r, m), q)\right) \\
& =1+\sum_{m \geq 1} \frac{1}{r^{m} m !} \sum_{y \in W(r, m)} P(y, T(r, m), q) p_{y} .
\end{aligned}
$$

In the following result $\mu(d)$ denotes the Möbius function. 
Theorem 3.1. If $R_{r, i, \theta}:=\sum_{d \mid i}\left|\left\{\zeta \in \mu_{r}: \zeta^{d}=\theta\right\}\right| \mu(d)\left(q^{i / d}-1\right) \in \mathbb{C}[q]$,

$$
P(r, q)=\prod_{\substack{i \geq 1 \\ \theta \in \mu_{r}}}\left(1+p_{i}(\theta)\right)^{R_{r, i, \theta} / r i} .
$$

PROOF. This follows from Hanlon's result [4, Corollary 2.3] on the Möbius functions of Dowling lattices. Within the reflection group context, it follows from results of Lehrer in [8] $(r=1)$, [7] $(r=2)$ and [1,9] (general $r$ ). A short proof for all $r \geq 2$, based on an 'equivariant inclusion-exclusion' argument of Getzler, is given in [5, Theorem 8.4] $(T(r, m)$ is the same as what is there called $M(r, m))$. The $r=1$ case can be proved by the same method (note that $T(1, m)$ is different from the variety $M(1, m)$ considered in [5, Theorem 8.2], since it has the extra condition of nonzero coordinates).

Recovering the traces of individual elements by the above rule, we get an equivalent statement, closer to Hanlon's and Lehrer's: if $y$ in $W(r, m)$ has $a_{i}(\zeta)$ cycles of length $i$ and type $\zeta$,

$$
P(y, T(r, m), q)=\prod_{\substack{i \geq 1 \\ \zeta \in \mu_{r}}} R_{r, i, \zeta}\left(R_{r, i, \zeta}-r i\right) \cdots\left(R_{r, i, \zeta}-\left(a_{i}(\zeta)-1\right) r i\right) .
$$

There is an alternative description of the polynomials $R_{r, i, \theta}$. Define

$$
R_{i}^{(r)}:=\sum_{\substack{d \mid i \\ \operatorname{gcd}(d, r)=1}} \mu(d)\left(q^{i / d}-1\right) \in \mathbb{C}[q] .
$$

LEMMA 3.2. If $t(\theta)$ denotes the order of $\theta$,

$$
R_{r, i, \theta}=\sum_{s \mid \operatorname{gcd}(r / t(\theta), i)} s \mu(s) R_{i / s}^{(r)} .
$$

PROOF. Since $\mu_{r}$ is cyclic of order $r$,

$$
\left|\left\{\zeta \in \mu_{r}: \zeta^{d}=\theta\right\}\right|= \begin{cases}\operatorname{gcd}(d, r) & \text { if } \operatorname{gcd}(d, r) \mid(r / t(\theta)) \\ 0 & \text { otherwise }\end{cases}
$$

Hence

$$
R_{r, i, \theta}=\sum_{s \mid(r / t(\theta))} s \sum_{\substack{d \mid i \\ \operatorname{gcd}(d, r)=s}} \mu(d)\left(q^{i / d}-1\right) .
$$

The sum over $d$ has no terms unless $s \mid i$, in which case it equals

$$
\sum_{\substack{d \mid(i / s) \\ \operatorname{gcd}(d, r)=1}} \mu(d s)\left(q^{i / d s}-1\right) .
$$

Since $\operatorname{gcd}(d, r)=1$ implies $\mu(d s)=\mu(d) \mu(s)$, this is $\mu(s) R_{i / s}^{(r)}$. 
This lemma makes it clear that the $r=2$ case of (3.1) is indeed equivalent to [7, Theorem 5.6].

As for $\mathbb{P} T(r, m)$, we have that, for all $y \in W(r, m)$,

$$
P(y, \mathbb{P} T(r, m), q)=\frac{1}{q-1} P(y, T(r, m), q) .
$$

For this, one need only show that the isomorphism

$$
\varphi: T(r, m) \rightarrow \mathbb{P} T(r, m) \times \mathbb{C}^{\times}:\left(z_{1}, \ldots, z_{m}\right) \mapsto\left(\left[z_{1}: \cdots: z_{m}\right], z_{1}\right)
$$

induces a $W(r, m)$-equivariant map on cohomology. It is enough to check that $w \circ \varphi$ and $\varphi \circ w$ are homotopic for all $w$ in a set of generators for $W(r, m)$, which is straightforward.

Now, for any $\chi \in \widehat{\mu_{n}}$, define the generating function

$$
\begin{aligned}
P(\chi, q) & :=1+\sum_{m \geq 1} \operatorname{ch}_{S_{m}}\left(P^{S_{m}}\left(\chi^{-1}, T^{(n)}(1, m), q\right)\right) \\
& =1+\sum_{m \geq 1} \frac{1}{m !} \sum_{w \in S_{m}} P\left(w, \chi^{-1}, T^{(n)}(1, m), q\right) p_{w} \in \mathbb{A}(1)[q]
\end{aligned}
$$

We want a formula for this similar to Theorem 3.1. Define

$$
P_{i}^{(r)}:=\prod_{s \mid \operatorname{gcd}(r, i)}\left(1-\left(-p_{i}\right)^{r / s}\right)^{s \mu(s) R_{i / s}^{(r)} / r i} \in \mathbb{A}(1)[q] .
$$

THEOREM 3.3. If $\chi \in \widehat{\mu_{n}}$ has $\operatorname{order} r, P(\chi, q)=\prod_{i \geq 1} P_{i}^{(r)}$.

Proof. Using Proposition 2.2, we see that $P(\chi, q)$ equals

$$
1+\sum_{m \geq 1} \frac{1}{n^{m} m !} \sum_{\substack{w \in S_{m} \\\left(\zeta_{i}\right) \in \mu_{n}^{m}}} \chi\left(\zeta_{1} \cdots \zeta_{m}\right) P\left(w\left(\zeta_{1}, \ldots, \zeta_{m}\right), T(n, m), q\right) p_{w}
$$

which is precisely the result of applying to $P(n, q)$ the specialization $p_{i}(\theta) \rightarrow \chi(\theta) p_{i}$. 
So, by Theorem 3.1,

$$
\begin{aligned}
P(\chi, q) & =\exp \sum_{\substack{i \geq 1 \\
\theta \in \mu_{n}}} \frac{R_{n, i, \theta}}{n i} \log \left(1+\chi(\theta) p_{i}\right) \\
& =\exp \sum_{\substack{i \geq 1 \\
\theta \in \mu_{n} \\
m \geq 1}} \frac{-R_{n, i, \theta}}{n m i} \chi(\theta)^{m}\left(-p_{i}\right)^{m} \\
& =\exp \sum_{\substack { i \geq 1 \\
\begin{subarray}{c}{\zeta \in \mu_{n} \\
m \geq 1{ i \geq 1 \\
\begin{subarray} { c } { \zeta \in \mu _ { n } \\
m \geq 1 } }\end{subarray}} \frac{-\mu(d)}{n m i} \chi(\zeta)^{d m}\left(q^{i / d}-1\right)\left(-p_{i}\right)^{m} \\
& =\exp \sum_{\substack{i \geq 1 \\
d|i \\
m \geq 1, r| d m}} \frac{-\mu(d)}{m i}\left(q^{i / d}-1\right)\left(-p_{i}\right)^{m},
\end{aligned}
$$

since $\sum_{\zeta \in \mu_{n}} \chi(\zeta)^{d m}=n$ if $\chi^{d m}$ is trivial, and vanishes otherwise. Thus we need only show that

$$
P_{i}^{(r)}=\exp \sum_{\substack{d|i \\ m \geq 1, r| d m}} \frac{-\mu(d)}{m i}\left(q^{i / d}-1\right)\left(-p_{i}\right)^{m} .
$$

The condition $r \mid d m$ is equivalent to $(r / \operatorname{gcd}(d, r)) \mid m$. Writing $s$ for $\operatorname{gcd}(d, r)$, the right-hand side becomes

$$
\begin{aligned}
& \exp \sum_{\substack{s|r \\
d| i, \operatorname{gcd}(d, r)=s \\
m \geq 1,(r / s) \mid m}} \frac{-\mu(d)}{m i}\left(q^{i / d}-1\right)\left(-p_{i}\right)^{m} \\
& \quad=\exp \sum_{s \mid \operatorname{gcd}(r, i)} \frac{s}{r i} \log \left(1-\left(-p_{i}\right)^{r / s}\right) \sum_{\substack{d \mid i \\
\operatorname{gcd}(d, r)=s}} \mu(d)\left(q^{i / d}-1\right) .
\end{aligned}
$$

By the same argument as in the proof of Lemma 3.2, the sum over $d$ equals $\mu(s) R_{i / s}^{(r)}$ as required.

Note that $P(\chi, q)$ depends only on $r$, not on $n$ or $\chi$, and that, as predicted in Remark 2.1, its nonzero homogeneous components all have degree divisible by $r$.

There is no formula as neat as (3.1) for the individual polynomials $P\left(w, \chi^{-1}, T^{(n)}(1, m), q\right)$. However, if $w \in S_{m}$ has $a_{i}$ cycles of length $i$, we know that

$$
P\left(w, \chi^{-1}, T^{(n)}(1, m), q\right)=\prod_{i \geq 1} a_{i} ! i^{a_{i}}\left(\text { coefficient of } p_{i}^{a_{i}} \text { in } P_{i}^{(r)}\right)
$$


Note that, for the right-hand side to be nonzero, $a_{i}$ must be divisible by $(r / \operatorname{gcd}(r, i))$ for all $i$. In the special case that $\operatorname{gcd}(r, i)=1$,

$$
P_{i}^{(r)}=\left(1-\left(-p_{i}\right)^{r}\right)^{R_{i}^{(r)} / r i},
$$

and the coefficient of $p_{i}^{a_{i}}$, where $a_{i}$ is divisible by $r$, is

$$
(-1)^{a_{i}-a_{i} / r} \frac{R_{i}^{(r)}\left(R_{i}^{(r)}-r i\right)\left(R_{i}^{(r)}-2 r i\right) \cdots\left(R_{i}^{(r)}-\left(a_{i}-r\right) i\right)}{(r i)^{a_{i} / r}\left(a_{i} / r\right) !} .
$$

Now consider some further special cases. The $r=1$ case of Theorem 3.3 says that

$$
P(\operatorname{triv}, q)=\prod_{i \geq 1}\left(1+p_{i}\right)^{R_{i}^{(1)} / i}=P(1, q),
$$

reflecting the fact that the quotient of $T^{(n)}(1, m)$ by $\mu_{n}$ is $T(1, m)$. Slightly more interesting is the $r=2$ case. We have

$$
P_{i}^{(2)}= \begin{cases}\left(1-p_{i}^{2}\right)^{R_{i}^{(2)} / 2 i} & \text { if } i \text { is odd } \\ \left(1-p_{i}^{2}\right)^{R_{i}^{(2)} / 2 i}\left(1+p_{i}\right)^{-R_{i / 2}^{(2)} / i} & \text { if } i \text { is even. }\end{cases}
$$

Hence if $i$ is even, the coefficient of $p_{i}^{a_{i}}$ is

$$
\sum_{j=0}^{\left\lfloor a_{i} / 2\right\rfloor}(-1)^{j}\left(\begin{array}{c}
R_{i}^{(2)} / 2 i \\
j
\end{array}\right)\left(\begin{array}{c}
-R_{i / 2}^{(2)} / i \\
a_{i}-2 j
\end{array}\right)
$$

Returning to Problem 1.1, (2.1) and (3.4) tell us that, if $w \in S_{n}$ has $a_{i}$ cycles of length $i$,

$$
P(w, \chi, S T(1, n), q)=\frac{1}{q-1} \prod_{i \geq 1} a_{i} ! i^{a_{i}}\left(\text { coefficient of } p_{i}^{a_{i}} \text { in } P_{i}^{(r)}\right) .
$$

Since there are $\phi(r)$ characters $\chi \in \widehat{\mu_{n}}$ of order $r$, we deduce that

$$
P(w, S T(1, n), q)=\frac{1}{q-1} \sum_{r \mid n} \phi(r) \prod_{i \geq 1} a_{i} ! i^{a_{i}}\left(\text { coefficient of } p_{i}^{a_{i}} \text { in } P_{i}^{(r)}\right) .
$$

REMARK 3.4. As mentioned in the introduction, if $q$ is specialized to a prime power congruent to $1 \bmod n$, the right-hand side of (3.7) equals the formula given in [3, Theorem 5.8] for the number of $\mathbb{F}_{q}$-points of the regular set of a maximal torus of $S L_{n}\left(\mathbb{F}_{q}\right)$ obtained from a maximally split one by twisting with $w$. (To see this, use the expression (3.3) for $P_{i}^{(r)}$; the coefficient of $p_{i}^{a_{i}}$ is called $R_{a_{i}, n}^{i}(q)$ in [3].) This is no surprise: general principles imply that for all but finitely many primes,

$$
P(w, S T(1, n), q)=\left|S T(1, n)\left(\overline{\mathbb{F}_{q}}\right)^{w F}\right| .
$$

See [2, Section 5.3, Example 5.6] for the details. 


\section{Induction}

We now aim to prove Theorem 1.2 by interpreting the generating function $P(\chi, q)$ in terms of induced characters. Recall that $W(r, m)$ can be embedded in $S_{r m}$ as the centralizer of the product of $m$ disjoint $r$-cycles. For any $\theta \in \mu_{r}$, let $t(\theta)$ denote the order of $\theta$.

LEMMA 4.1. For any $W(r, m)$-module $M$,

$$
\operatorname{ch}_{S_{r m}}\left(\left[\operatorname{Ind}_{W(r, m)}^{S_{r m}}(M)\right]\right)=\left.\operatorname{ch}_{W(r, m)}([M])\right|_{p_{i}(\theta) \rightarrow p_{i t(\theta)}^{r / t(\theta)}} .
$$

PROOF. This is a direct consequence of Frobenius' formula for induced characters, once one observes that a cycle of length $i$ and type $\theta$ in $W(r, m)$ becomes the product of $r / t(\theta)$ disjoint $i t(\theta)$-cycles when regarded as an element of $S_{r m}$.

LEMMA 4.2. For any $W(r, m)$-module $M$,

$$
\begin{aligned}
& \operatorname{ch}_{S_{r m}}\left(\left[\varepsilon_{r m}\right.\right.\left.\left.\otimes \operatorname{Ind}_{W(r, m)}^{S_{r m}}\left(\operatorname{det}_{m}^{-1} \otimes M\right)\right]\right) \\
& \quad=\left.(-1)^{r m-m} \operatorname{ch}_{W(r, m)}([M])\right|_{p_{i}(\theta) \rightarrow-\theta^{-1}\left(-p_{i t(\theta)}\right)^{r / t(\theta)} .}
\end{aligned}
$$

PROOF. If $y \in W(r, i)$ is a cycle of length $i$ and type $\theta$,

$$
\varepsilon_{r i}(y) \operatorname{det}_{i}(y)^{-1}=(-1)^{i-1+(i t(\theta)-1) r / t(\theta)} \theta^{-1}=(-1)^{i-1+r i-r / t(\theta)} \theta^{-1} .
$$

Also, if $y \in W(r, m)$,

$$
\left.p_{y}\right|_{p_{i}(\theta) \rightarrow(-1)^{r i+i} p_{i}(\theta)}=(-1)^{r m-m} p_{y} .
$$

Hence

$$
\begin{aligned}
\operatorname{ch}_{W(r, m)} & \left(\left[\varepsilon_{r m} \otimes \operatorname{det}_{m}^{-1} \otimes M\right]\right) \\
& =\left.(-1)^{r m-m} \operatorname{ch}_{W(r, m)}([M])\right|_{p_{i}(\theta) \rightarrow-\theta^{-1}(-1)^{r / t(\theta)}} p_{i}(\theta)
\end{aligned},
$$

and the result follows by applying the previous lemma.

Now define an element $P^{\prime}(r, q) \in \mathbb{A}(1)[q]$ by

$$
\begin{aligned}
& P^{\prime}(r, q):=\left.P(r, q)\right|_{p_{i}(\theta) \rightarrow-\theta^{-1}\left(-p_{i t(\theta)}\right)^{r / t(\theta)}} \\
& \quad=1+\left.\sum_{m \geq 1} \operatorname{ch}_{W(r, m)}\left(P^{W(r, m)}(T(r, m), q)\right)\right|_{p_{i}(\theta) \rightarrow-\theta^{-1}\left(-p_{i t(\theta)}\right)^{r / t(\theta)}} \\
& \quad=1+\sum_{m \geq 1}(-1)^{r m-m} \operatorname{ch}_{S_{r m}}\left(\varepsilon_{r m} \otimes \operatorname{Ind}_{W(r, m)}^{S_{r m}}\left(\operatorname{det}_{m}^{-1} \otimes P^{W(r, m)}(T(r, m), q)\right)\right) .
\end{aligned}
$$

Proposition 4.3. $P^{\prime}(r, q)=\prod_{i \geq 1} P_{i}^{(r)}$. 
PROOF. By Theorem 3.1 and Lemma 3.2,

$$
\begin{aligned}
P^{\prime}(r, q)= & \prod_{\substack{i \geq 1 \\
\theta \in \mu_{r}}}\left(1-\theta^{-1}\left(-p_{i t(\theta)}\right)^{r / t(\theta)}\right)^{R_{r, i, \theta} / r i} \\
= & \prod_{\substack{i \geq 1 \\
\theta \in \mu_{r} \\
s \mid \operatorname{gcd}(r / t(\theta), i)}}\left(1-\theta^{-1}\left(-p_{i t(\theta)}\right)^{r / t(\theta)}\right)^{s \mu(s) R_{i / s}^{(r)} / r i} .
\end{aligned}
$$

Applying to this the Möbius inversion formula for cyclotomic polynomials, in the form

$$
\prod_{\substack{\theta \in \mu_{r} \\ t(\theta)=t}}\left(1-\theta^{-1} X\right)=\prod_{u \mid t}\left(1-X^{t / u}\right)^{\mu(u)},
$$

we obtain

$$
P^{\prime}(r, q)=\prod_{\substack{i \geq 1 \\ t|r \\ s| \operatorname{gcd}(r / t, i) \\ u \mid t}}\left(1-\left(-p_{i t}\right)^{r / u}\right)^{s \mu(s) \mu(u) R_{i / s}^{(r)} / r i}
$$

Write this as $\prod_{i \geq 1} Q_{i}^{(r)}$, where $Q_{i}^{(r)}$ is the product of all factors involving the variable $p_{i}$. Thus

$$
\begin{aligned}
Q_{i}^{(r)} & =\exp \sum_{\substack{t|\operatorname{gcd}(r, i) \\
s| \operatorname{gcd}(r / t, i / t) \\
u \mid t}} \frac{s t \mu(s) \mu(u)}{r i} R_{i / s t}^{(r)} \log \left(1-\left(-p_{i}\right)^{r / u}\right) \\
& =\exp \sum_{\substack{v|\operatorname{gcd}(r, i) \\
u| v \\
s \mid(v / u)}} \frac{v \mu(s) \mu(u)}{r i} R_{i / v}^{(r)} \log \left(1-\left(-p_{i}\right)^{r / u}\right),
\end{aligned}
$$

where we have set $v=s t$. Since $\sum_{s \mid(v / u)} \mu(s)$ is nonzero if and only if $u=v$, we find that $Q_{i}^{(r)}=P_{i}^{(r)}$ as required.

COROLlaRY 4.4. If $\chi \in \widehat{\mu_{n}}$ has order $r, P(\chi, q)=P^{\prime}(r, q)$.

PROOF. Combine Theorem 3.3 and Proposition 4.3.

COROLLARY 4.5. If $\chi \in \widehat{\mu_{n}}$ has order $r$, and $r \mid m$, we have the following equality in $R\left(S_{m}\right)[q]$ :

$$
\begin{aligned}
& P^{S_{m}}\left(\chi^{-1}, T^{(n)}(1, m), q\right) \\
& \quad=(-1)^{m-m / r} \varepsilon_{m} \otimes \operatorname{Ind}_{W(r, m / r)}^{S_{m}}\left(\operatorname{det}_{m / r}^{-1} \otimes P^{W(r, m / r)}(T(r, m / r), q)\right) .
\end{aligned}
$$


PROOF. Under the isomorphism $\mathrm{ch}_{S_{m}}$, the left-hand side corresponds to the degree$m$ term of $P(\chi, q)$, and the right-hand side corresponds to the degree- $m$ term of $P^{\prime}(r, q)$.

To translate Corollary 4.5 into an isomorphism of $S_{m}$-modules, we take coefficients of $q^{i-m}$ on both sides and multiply by $(-1)^{i}$ to obtain

$$
H_{c}^{i}\left(T^{(n)}(1, m)\right)_{\chi^{-1}} \cong \varepsilon_{m} \otimes \operatorname{Ind}_{W(r, m / r)}^{S_{m}}\left(\operatorname{det}_{m / r}^{-1} \otimes H_{c}^{i-m+m / r}(T(r, m / r))\right) .
$$

By Poincaré duality, this is equivalent to

$$
H^{2 m-i}\left(T^{(n)}(1, m)\right)_{\chi} \cong \varepsilon_{m} \otimes \operatorname{Ind}_{W(r, m / r)}^{S_{m}}\left(\operatorname{det}_{m / r} \otimes H^{m / r+m-i}(T(r, m / r))\right),
$$

which after replacing $2 m-i$ by $i$ gives

$$
H^{i}\left(T^{(n)}(1, m)\right)_{\chi} \cong \varepsilon_{m} \otimes \operatorname{Ind}_{W(r, m / r)}^{S_{m}}\left(\operatorname{det}_{m / r} \otimes H^{i-m+m / r}(T(r, m / r))\right) .
$$

Finally, we prove Theorem 1.2. Equations (2.1), (3.2), and Corollary 4.5 together imply

$$
\begin{aligned}
P^{S_{n}}(\chi & , S T(1, n), q) \\
= & \frac{(-1)^{n-n / r}}{q-1} \varepsilon_{n} \otimes \operatorname{Ind}_{W(r, n / r)}^{S_{n}}\left(\operatorname{det}_{n / r}^{-1} \otimes P^{W(r, n / r)}(T(r, n / r), q)\right) \\
& =(-1)^{n-n / r} \varepsilon_{n} \otimes \operatorname{Ind}_{W(r, n / r)}^{S_{n}}\left(\operatorname{det}_{n / r}^{-1} \otimes P^{W(r, n / r)}(\mathbb{P} T(r, n / r), q)\right) .
\end{aligned}
$$

Taking coefficients of $q^{i-n+1}$ on both sides and multiplying by $(-1)^{i}$, we get an isomorphism of $S_{n}$-modules:

$$
H_{c}^{i}(S T(1, n))_{\chi} \cong \varepsilon_{n} \otimes \operatorname{Ind}_{W(r, n / r)}^{S_{n}}\left(\operatorname{det}_{n / r}^{-1} \otimes H_{c}^{i-n+n / r}(\mathbb{P} T(r, n / r))\right) .
$$

Since the right-hand side depends only on $n$ and the order of $\chi$, this remains true if $\chi$ is replaced by $\chi^{-1}$. Then Theorem 1.2 follows by Poincaré duality.

\section{References}

[1] J. Blair and G. I. Lehrer, 'Cohomology actions and centralisers in unitary reflection groups', Proc. London Math. Soc. (3) 83(3) (2001), 582-604.

[2] A. Dimca and G. I. Lehrer, 'Purity and equivariant weight polynomials', in: Algebraic groups and Lie groups, Australian Mathematical Society Lecture Series, 9 (Cambridge University Press, Cambridge, 1997), pp. 161-181.

[3] P. Fleischmann and I. Janiszczak, 'The number of regular semisimple elements for Chevalley groups of classical type', J. Algebra 155 (1993), 482-528.

[4] P. Hanlon, 'The characters of the wreath product group acting on the homology groups of the Dowling lattices', J. Algebra 91 (1984), 430-463. 
[5] A. Henderson, 'Representations of wreath products on cohomology of De Concini-Procesi compactifications', Int. Math. Res. Not. 2004(20) (2004), 983-1021.

[6] , 'Bases for certain cohomology representations of the symmetric group', J. Algebraic Combin. 24(4) (2006), 361-390.

[7] G. I. Lehrer, 'On hyperoctahedral hyperplane complements', in: The Arcata Conf. on representations of finite groups (Arcata, CA, 1986), Proceedings of Symposia in Pure Mathematics, 47 (American Mathematical Society, Providence, RI, 1987), pp. 219-234.

[8] _ - 'On the Poincaré series associated with Coxeter group actions on complements of hyperplanes', J. London Math. Soc. (2) 36 (1987), 275-294.

[9] - 'Poincaré polynomials for unitary reflection groups', Invent. Math. 120 (1995), 411-425.

[10] I. G. Macdonald, Symmetric functions and Hall polynomials, 2nd edn (Oxford University Press, Oxford, 1995).

ANTHONY HENDERSON, School of Mathematics and Statistics, University of Sydney, NSW 2006, Australia

e-mail: anthonyh@maths.usyd.edu.au 